\title{
An Initial and Boundary Value Problem Modeling Fish-like Swimming
}

\author{
JorGE SAN MARTÍN, \\ Departamento de Ingeniería Matemática, Universidad de Chile, \\ Casilla 170/3-Correo 3, Santiago, Chile. \\ (jorge@dim.uchile.cl). \\ Jean-François Scheid, Takéo Takahashi, Marius Tucsnak \\ Institut Élie Cartan UMR 7502, Nancy-Université, CNRS, INRIA, \\ BP239, 54506 Vandouvre-lès-Nancy, Cedex, France. \\ (Jean-Francois.Scheid@iecn.u-nancy.fr, \\ Takeo.Takahashi@iecn.u-nancy.fr, \\ Marius. Tucsnak@iecn.u-nancy.fr). \\ and \\ INRIA Lorraine, Projet CORIDA.
}

July 27, 2006

\begin{abstract}
In this paper we consider an initial and boundary value problem modeling the self-propelled motion of solids in a bi-dimensional viscous incompressible fluid. The self-propelling mechanism, consisting in appropriate deformations of the solids, is a simplified model for the propulsion mechanism of fish-like swimmers. The governing equations are composed of the Navier-Stokes equations for the fluid, coupled to Newton's laws for the solids. Since we consider the case in which the fluid-solid systems fills a bounded domain we have to tackle a free boundary value problem. The main theoretical result in the paper asserts the global existence and uniqueness (up to possible contacts) of strong solutions of this problem. The second novelty brought in by this work is that we give a numerical method for the fluid-solid system. This method allows the simulation of the simultaneous displacement of several swimmers and it is tested on several examples.
\end{abstract}

\section{Introduction}

Understanding the locomotion mechanism of aquatic animals is a problem which has intrigued researchers for a long time. The first treatise on this subject is Borelli [1] which was first published in 1680 and 1681. Much later (from 1932 to 1936), a detailed study of the kinematics of fish-like swimming has been performed in a series of papers of the zoologist James Gray. In Gray [17] it has been shown that the swimming mechanism of the eel is based on an undulatory deformation of its body and some of the parameters of 
this deformation have been determined. In the still controversial paper Gray [16], it was estimated that the swimming efficiency of a dolphin is much larger than the efficiency of conventional propellers for underwater vehicles.

More mathematically oriented scientists became involved in understanding fish motion after 1950 (see Taylor [32] for an early work in this direction). Further progress on the mathematical modeling of fish swimming have been reported in the books of Lighthill [24], Childress [6] and in several articles (see, for instance, Wu [35], Sparenberg [30] and references therein).

More recently, researchers have been interested to build, study and control biomimetic underwater vehicles which move by changes of shape and not by direct propulsion, such as with propellers. The work in this direction has been initiated in Tryantafyllou and Tryantafyllou [34] and developed in an important number of papers which tackled both theoretical and experimental issues (see, for instance, Kelly and Murray [21], Kanso, Marsden, Rowley and Melli-Huber [20] and references therein).

In all the papers mentioned above, the fluid is supposed to be inviscid and in irrotational flow. This assumption implies that, provided that we have solved an elliptic boundary value problem, the model reduces to the ordinary differential equations coming from Newton's laws. If we do not neglect the viscosity effects, the relevant model consists in the non stationary Navier-Stokes equations for the fluid which are coupled to Newton's laws for the fish-like swimming object. As far as we know, only few papers have tackled the problem from this perspective (see Carling [4], Liu and Kawachi [26]). These papers assume that the fluid-body systems fills the whole space and they introduce computational fluid dynamics methods to investigate the locomotion of fish-like objects in a viscous incompressible fluid. The numerical methods introduced in the above mentioned papers do not seem adapted to the simultaneous simulation of the motion of several immersed objects. On the other hand, the mathematical analysis of the associated system of PDE's (that is existence, uniqueness and stability properties) seems an open problem.

In this paper we consider an initial and boundary value problem for a simplified model of fish-like swimming. This model consists in a solid undergoing an undulatory deformation, which is immersed in a viscous incompressible fluid. In the remaining part of this paper the undulating body is called the creature. The displacement of the creature is decomposed into a rigid part and a deformation (undulatory) part. The rigid part of the displacement results from the interaction of the fluid and the solid, whereas the deformation part is given. Since our aim is to possibly consider several creatures and to tackle the case of a bounded fluid-body system, the domain filled by the fluid is one of the unknowns. Therefore we have to tackle a free boundary value problem. This difficulty has been recently addressed in several papers for the case of rigid bodies immersed in a viscous incompressible fluid (see, for instance, Desjardins and Esteban [12], San Martín, Starovoitov and Tucsnak [29], Feireisl [13] or Gunzburger, Lee and Seregin [18]). Let us also mention that in the case of a fluid-rigid system filling the whole space the existence of self-propelled motions has been investigated in Galdi [14] and [15]. Recently, several works addressed the mathematical analysis of the interaction of a viscous incompressible fluid with an elastic or viscoelastic body (see, for instance Coutand and Shkoller [7], [8], Chambolle, Desjardins, Esteban and Grandmont [5], Desjardins, Esteban, Grandmont and Le Tallec [11] and Boulakia [3]). As it will be explained more precisely below, one of the particularities of our work is that we do not use any particular constitutive law for the solid but we impose the nonrigid part of the deformation. The advantages of this approach consist in the global character (up to 
possible contacts) of the obtained strong solutions and in the possibility of using numerical methods inspired by those developed in the rigid-fluid case.

Our main theoretical result asserts that the initial and boundary value problem obtained by coupling the Navier-Stokes equations for the fluid to Newton's law for the creature is well-posed in Sobolev type spaces. Our method is based on a change of variables introduced in Takahashi [31] combined to an appropriate lifting of the non rigid part of the velocity field and to some a priori estimates. Our method for proving the existence result can be extended to the case of several immersed bodies.

The second contribution brought in by this work consists in giving a weak formulation (of mixed type) and an approximation scheme for the governing equations. This scheme is finally tested on some undulatory motions observed by the zoologists in order to get straight-line-swimming or turning. Moreover, we take advantage of the fact that our method still works if we have several immersed bodies, in order to simulate the simultaneous swimming of two fish-like creatures.

This paper is organized as follows. In Section 2 we describe the model. Section 3 is devoted to the statement of the main theoretical result. The local existence of strong solutions is proved in Section 4. The proof of the global existence and uniqueness result is given in Section 5. In Section 6 we derive a weak form of the governing equations and we describe a semi-discretization with respect to the time variable. Finally, in Section 7 we describe a class of possible deformations of the swimmers and we illustrate them by numerical simulations.

\section{The governing equations}

\subsection{A kinematic model of fish-like swimming}

Denote by $\mathcal{S}_{0}$ the domain occupied by the creature in a reference configuration. We assume that $\mathcal{S}_{0}$ is an open connected set with $C^{\infty}$ boundary. Moreover, we suppose that the motion $X$ of $\mathcal{S}_{0}$ is a smooth mapping

$$
X: \mathcal{S}_{0} \times[0, \infty) \rightarrow \mathbb{R}^{2}
$$

which satisfies

$$
X(y, t)=X^{R}\left(X^{*}(y, t), t\right) \quad \forall y \in \mathcal{S}_{0}, \quad t \geqslant 0,
$$

where $X^{R}$ is a rigid displacement and $X^{*}$ is an appropriate smooth mapping, representing the undulatory deformation of the creature. In the remaining part of this work the rigid motion $X^{R}$ will be an unknown to be determined from the governing equations below, whereas the undulatory motion $X^{*}$ will be supposed to be known and to satisfy several assumptions which will be given in this section. An example of undulatory motion $X^{*}$ coming from zoological observations and satisfying our assumptions will be given in Section 7 .

Let us give the assumptions on the undulatory motion $X^{*}$. We choose a system of coordinates with the origin in the mass center of $\mathcal{S}_{0}$. Our first assumption is

(H1) For every $t \geqslant 0$, the mapping $y \mapsto X^{*}(y, t)$ is a $C^{\infty}$ diffeomorphism from $\overline{\mathcal{S}}_{0}$ onto 
$\overline{\mathcal{S}}^{*}(t)$, where $\mathcal{S}^{*}(t)=X^{*}\left(\mathcal{S}_{0}, t\right)$. Moreover, for every $y \in \mathcal{S}_{0}$, the mapping $t \mapsto X^{*}(y, t)$ is of class $C^{\infty}$.

For every $t \geqslant 0$ we denote by $Y^{*}$ the inverse de $X^{*}$, i.e. the diffeomorphism satisfying

$$
X^{*}\left(Y^{*}\left(x^{*}, t\right), t\right)=x^{*}, \quad Y^{*}\left(X^{*}(y, t), t\right)=y
$$

for every $x^{*} \in \mathcal{S}^{*}(t), t \geqslant 0$ and $y \in \mathcal{S}_{0}$.

The deformation $X^{*}$ is also supposed to satisfy the following assumption.

(H2) The total volume of the creature is preserved, i.e.,

$$
\int_{\mathcal{S}^{*}(t)} \mathrm{d} x^{*}=\int_{\mathcal{S}_{0}} \mathrm{~d} y \quad \forall t \geqslant 0
$$

Denote by $w^{*}$ the undulatory velocity of creature, written as a vector field on $\mathcal{S}^{*}(t)$, i.e.,

$$
w^{*}\left(x^{*}, t\right)=\left.\frac{\partial X^{*}}{\partial t}(y, t)\right|_{y=Y^{*}\left(x^{*}, t\right)} \quad \forall x^{*} \in \mathcal{S}^{*}(t), \quad t \geqslant 0 .
$$

Let $\rho_{0}$ be the density field of the solid in the reference configuration $\mathcal{S}_{0}$ and let $\rho^{*}(t)$ be the density field of $\mathcal{S}^{*}(t)$. The mass conservation principle applied to the whole body gives

$$
\int_{\mathcal{S}^{*}(t)} \rho^{*}\left(x^{*}, t\right) \mathrm{d} x^{*}=\int_{\mathcal{S}_{0}} \rho_{0}(y) \mathrm{d} y \quad \forall t \geqslant 0,
$$

whereas the local form of the conservation of mass yields

$$
\rho^{*}\left(x^{*}, t\right)=\frac{\rho_{0}\left(Y^{*}\left(x^{*}, t\right)\right)}{\operatorname{det}\left(\nabla X^{*}\right)\left(Y^{*}\left(x^{*}, t\right)\right)} \quad \forall t \geqslant 0, \quad \forall x^{*} \in \mathcal{S}^{*}(t) .
$$

Moreover since our aim is to study self-propelled motions, it is natural to assume that the undulatory motion does not modify the linear and the angular momenta of the creature. By using the fact that the origin of our system of coordinates coincides with the mass center of $\mathcal{S}_{0}$, these assumptions can be written as

$$
\int_{\mathcal{S}^{*}(t)} \rho^{*}\left(x^{*}, t\right) w^{*}\left(x^{*}, t\right) \mathrm{d} x^{*}=0 \quad \text { for all } t \geqslant 0
$$

$$
\int_{\mathcal{S}^{*}(t)} \rho^{*}\left(x^{*}, t\right) x^{* \perp} \cdot w^{*}\left(x^{*}, t\right) \mathrm{d} x^{*}=0 \quad \text { for all } t \geqslant 0, \text { where, for } x=\left(\begin{array}{l}
x_{1} \\
x_{2}
\end{array}\right) \text {, we }
$$

denote by $x^{\perp}$ the vector $x^{\perp}=\left(\begin{array}{r}-x_{2} \\ x_{1}\end{array}\right)$.

Remark 2.1. Assumption (H3) implies, in particular, that the position of the center of mass of the body is not affected by the undulatory motion, i.e. that :

$$
\int_{\mathcal{S}^{*}(t)} \rho^{*}\left(x^{*}, t\right) x^{*} \mathrm{~d} x^{*}=0 \quad \forall t \geqslant 0
$$


The rigid motion $X^{R}: \mathbb{R}^{2} \times[0, \infty) \rightarrow \mathbb{R}^{2}$ is given by

$$
X^{R}\left(x^{*}, t\right)=R_{\theta(t)} x^{*}+\xi(t),
$$

where $\xi:[0, \infty) \rightarrow \mathbb{R}^{2}$ is the trajectory of the mass center of the creature and, for every $t \geqslant 0, \theta(t)$ is an angle giving the orientation of the creature and $R_{\theta}$ is the matrix associated to the rotation of angle $\theta$. We stress that the functions $\xi$ and $\theta$ above are unknowns in our model. From the above it follows that the position of the creature at instant $t$ is given by

$$
\mathcal{S}(\xi(t), \theta(t), t)=R_{\theta(t)} \mathcal{S}^{*}(t)+\xi(t) \quad \forall t \geqslant 0 .
$$

It is easy to check that the Eulerian velocity field of the solid is given, for every $t \geqslant 0$ by

$$
u_{S}(x, t)=\xi^{\prime}(t)+\theta^{\prime}(t)(x-\xi(t))^{\perp}+w(x, t) \quad \forall x \in \mathcal{S}(\xi(t), \theta(t), t),
$$

where

$$
w(x, t)=R_{\theta(t)} w^{*}\left(R_{-\theta(t)}(x-\xi(t)), t\right) \quad \forall x \in \mathcal{S}(\xi(t), \theta(t), t) .
$$

In other words, the Eulerian velocity field of the creature can be decomposed in a part coming from the rigid motion (the first two terms in the right hand side of (2.7)) and a part $w$ coming from the deformation of the creature.

The Eulerian density field of the body is given by

$$
\rho(x, t)=\rho^{*}\left(R_{-\theta(t)}(x-\xi(t)), t\right) \quad \forall t \geqslant 0, \quad x \in \mathcal{S}(\xi(t), \theta(t), t)
$$

with $\rho^{*}$ given by (2.5). The mass $m$ of the body and its moment of inertia, with respect to an axis orthogonal to the plane of the motion and passing by the mass center of $\mathcal{S}(\xi(t), \theta(t), t)$, are as usually given by

$$
\begin{gathered}
m=\int_{\mathcal{S}(\xi(t), \theta(t), t)} \rho(x, t) \mathrm{d} x . \\
I(t)=\int_{\mathcal{S}(\xi(t), \theta(t), t)} \rho(x, t)|x-\xi(t)|^{2} \mathrm{~d} x .
\end{gathered}
$$

Let us notice that from (2.4), (2.9) and (2.10), we have that

$$
m=\int_{\mathcal{S}_{0}} \rho_{0}(y) \mathrm{d} y .
$$

\subsection{The full system}

Let $\Omega$ be an open bounded set in $\mathbb{R}^{2}$ representing the domain occupied by the solid-fluid system. Recalling that $\mathcal{S}(\xi(t), \theta(t), t)$ is the domain occupied by the solid at instant $t$, we have that the fluid fills, at instant $t$, the domain $\mathcal{F}(\xi(t), \theta(t), t)=\Omega \backslash \overline{\mathcal{S}(\xi(t), \theta(t), t)}$. 
With the above notation and by supposing that the density of the fluid is equal to one, the full system describing the self-propelled motion of the creature can be written as

$$
\begin{aligned}
\frac{\partial u}{\partial t}-\nu \Delta u+(u \cdot \nabla) u+\nabla p=0 & \text { in } \mathcal{F}(\xi, \theta, t), t \in(0, T), \\
\operatorname{div} u=0 & \text { in } \mathcal{F}(\xi, \theta, t), t \in(0, T), \\
u=0 & \text { on } \partial \Omega, t \in(0, T), \\
u=\xi^{\prime}+\theta^{\prime}(x-\xi)^{\perp}+w & \text { on } \partial \mathcal{S}(\xi, \theta, t), t \in(0, T), \\
m \xi^{\prime \prime}=-\int_{\partial \mathcal{S}(\xi, \theta, t)} \sigma(u, p) n \mathrm{~d} \Gamma & \text { in }(0, T), \\
\left(I \theta^{\prime}\right)^{\prime}=-\int_{\partial \mathcal{S}(\xi, \theta, t)}(x-\xi)^{\perp} \cdot \sigma(u, p) n \mathrm{~d} \Gamma & \text { in }(0, T) .
\end{aligned}
$$

In the above system $\nu>0$ stands for the viscosity of the fluid, $u$ is the Eulerian velocity field of the fluid and $p$ denotes the pressure field of the fluid. A prime stands for the derivation operator with respect to time. By using the classical notation

$$
D(u)=\frac{1}{2}\left((\nabla u)+(\nabla u)^{T}\right),
$$

the stress tensor field $\sigma$ is defined by

$$
\sigma(u, p)=2 \nu D(u)-p \mathrm{Id},
$$

where Id is the identity matrix in $M_{2}(\mathbb{R})$. Moreover, for $t \in[0, T]$ and $x \in \partial \mathcal{S}(\xi(t), \theta(t), t)$ we denote by $n(x, t)$ the unit normal to $\mathcal{S}(\xi(t), \theta(t), t)$ oriented towards the solid. Recall that the mass $m$ and the moment of inertia $I(t)$ of the solid at instant $t$ are defined by (2.10) and (2.11).

The system (2.12)-(2.19) is completed by the initial conditions

$$
\begin{aligned}
u(x, 0)=u_{0}(x) & x \in \mathcal{F}(\xi(0), \theta(0), 0), \\
\xi(0)=\xi_{0}, \theta(0)=\theta_{0}, & \xi^{\prime}(0)=g_{0}, \theta^{\prime}(0)=\omega_{0} .
\end{aligned}
$$

Remark 2.2. Notice that in the balance of linear and angular momenta for the solid $\mathcal{S}(\xi(t), \theta(t), t)$ (see equations $(2.16)$ and $(2.17)$ ), there is no force or torque corresponding to the non rigid deformation of $\mathcal{S}(\xi(t), \theta(t), t)$. This is due to the fact that the non rigid deformation is produced only by forces which are internal to the creature and it is coherent with assumptions (H3) and (H4) which imply that the linear and angular momenta resulting from the undulatory motion are vanishing.

Remark 2.3. From assumption (H2) it follows that

$$
\int_{\partial \mathcal{S}(\xi, \theta, t)} w \cdot n \mathrm{~d} \Gamma=0
$$

which is compatible with the condition div $u=0$ in $\mathcal{F}(\xi, \theta, t)$. Moreover, since

$$
I(t)=\int_{\mathcal{S}^{*}(t)} \rho^{*}\left(x^{*}, t\right)\left|x^{*}\right|^{2} \mathrm{~d} x^{*}=\int_{\mathcal{S}_{0}} \rho_{0}(y)\left|X^{*}(y, t)\right|^{2} \mathrm{~d} y,
$$

it follows that there exists $I_{0}>0$ with $I(t) \geqslant I_{0}$ for all $t \in[0, T]$. 


\section{Statement of the existence and uniqueness result}

In the sequel, we denote $\mathcal{F}=\mathcal{F}(\xi(0), \theta(0), 0)$ and $\mathcal{S}=\mathcal{S}(\xi(0), \theta(0), 0)$. Without loss of generality, we can assume that $\xi(0)=\xi_{0}=0$, that $\theta(0)=\theta_{0}=0$, and that $\mathcal{S}^{*}(0)=\mathcal{S}_{0}$. In particular, we have that $\mathcal{S}=\mathcal{S}_{0}$. Moreover, if there is no risk of confusion, we denote

$$
\mathcal{S}(t)=\mathcal{S}(\xi(t), \theta(t), t), \quad \mathcal{F}(t)=\mathcal{F}(\xi(t), \theta(t), t) .
$$

In order to state the main result we need some function spaces. As usual, we denote by $H^{s}(\mathcal{F})$ the classical Sobolev space of order $s \in \mathbb{R}$ and

$$
\left.\mathcal{H}^{s}(\mathcal{F}(t))\right)=\left[H^{s}(\mathcal{F}(t))\right]^{2}, \quad \mathcal{H}^{s}(\mathcal{F})=\left[H^{s}(\mathcal{F})\right]^{2}, \quad \mathcal{H}^{s}(\Omega)=\left[H^{s}(\Omega)\right]^{2} .
$$

For $q \geqslant 1$ we set

$$
\left.\mathcal{L}^{q}(\mathcal{F}(t))\right)=\left[L^{q}(\mathcal{F}(t))\right]^{2}, \quad \mathcal{L}^{q}(\mathcal{F})=\left[L^{q}(\mathcal{F})\right]^{2}, \quad \mathcal{L}^{q}(\Omega)=\left[L^{q}(\Omega)\right]^{2} .
$$

Let $\xi \in H^{2}\left((0, T) ; \mathbb{R}^{2}\right), \theta \in H^{2}((0, T) ; \mathbb{R})$ be two functions such that $\overline{\mathcal{S}(\xi(t), \theta(t), t)} \subset \Omega$ for all $t \in[0, T]$ (recall that $\mathcal{S}(\xi(t), \theta(t), t)$ was defined in $(2.6)$ ). Let $\Psi: \mathbb{R}^{2} \times[0, T] \rightarrow \mathbb{R}^{2}$ be a $C^{\infty}$-diffeomorphism from $\mathcal{F}$ onto $\mathcal{F}(\xi(t), \theta(t), t)$ such that the derivatives

$$
\frac{\partial^{i+k_{1}+k_{2}} \Psi}{\partial t^{i} \partial y_{1}^{k_{1}} \partial y_{2}^{k_{2}}}, \quad i \leqslant 1, \quad k_{1} \geqslant 0, \quad k_{2} \geqslant 0
$$

exist and are continuous (the existence of such a function will be discussed in Section 4).

Let us set

$$
\mathcal{F}_{T}=\left\{(x, t) \in \mathbb{R}^{2} \times(0, T) ; x \in \mathcal{F}(t)\right\} .
$$

For $u \in\left[L^{2}\left(\mathcal{F}_{T}\right)\right]^{k}$, with $k \in\{1,2\}$, we denote by $u_{\Psi}$ the function

$$
u_{\Psi}: \mathcal{F} \times(0, T) \rightarrow \mathbb{R}^{2}, \quad(y, t) \mapsto u(\Psi(y, t), t) .
$$

We can now define the following functions spaces:

$$
\begin{aligned}
L^{2}\left(0, T ; \mathcal{H}^{2}(\mathcal{F}(t))\right) & =\left\{u \mid u_{\Psi} \in L^{2}\left(0, T ; \mathcal{H}^{2}(\mathcal{F})\right)\right\} \\
H^{1}\left(0, T ; \mathcal{L}^{2}(\mathcal{F}(t))\right) & =\left\{u \mid u_{\Psi} \in H^{1}\left(0, T ; \mathcal{L}^{2}(\mathcal{F})\right)\right\} \\
C\left([0, T] ; \mathcal{H}^{1}(\mathcal{F}(t))\right) & =\left\{u \mid u_{\Psi} \in C\left([0, T] ; \mathcal{H}^{1}(\mathcal{F})\right)\right\} \\
L^{2}\left(0, T ; H^{1}(\mathcal{F}(t))\right) & =\left\{p \mid p_{\Psi} \in L^{2}\left(0, T ; H^{1}(\mathcal{F})\right)\right\}
\end{aligned}
$$

Moreover, we set

$$
\begin{gathered}
\mathcal{U}(0, T ; \mathcal{F}(t))=L^{2}\left(0, T ; \mathcal{H}^{2}(\mathcal{F}(t))\right) \cap H^{1}\left(0, T ; \mathcal{L}^{2}(\mathcal{F}(t))\right) \cap C\left([0, T] ; \mathcal{H}^{1}(\mathcal{F}(t))\right), \\
\mathcal{U}(0, T ; \mathcal{F})=L^{2}\left(0, T ; \mathcal{H}^{2}(\mathcal{F})\right) \cap H^{1}\left(0, T ; \mathcal{L}^{2}(\mathcal{F})\right) \cap C\left([0, T] ; \mathcal{H}^{1}(\mathcal{F})\right) .
\end{gathered}
$$

Definition 3.1. Assume that $T>0$. A quadruplet $(u, p, \xi, \theta)$ with

$$
\begin{cases}u \in \mathcal{U}(0, T ; \mathcal{F}(t)), & p \in L^{2}\left(0, T ; H^{1}(\mathcal{F}(t))\right), \\ \xi \in\left[H^{2}(0, T)\right]^{2}, & \theta \in H^{2}(0, T),\end{cases}
$$

such that the distance from $\mathcal{S}(t)$ to $\partial \Omega$ is positive for all $t \in[0, T]$ and which satisfies (2.12)-(2.21) is called a strong solution of $(2.12)-(2.21)$ on $[0, T]$. 
The main theoretical result of this paper concerns the existence and uniqueness of strong solutions for the system (2.12)-(2.21) and it can be stated as follows.

Theorem 3.2. Assume that $X^{*}$ satisfies assumptions (H1)-(H4) above. Moreover, assume that $u_{0} \in \mathcal{H}^{1}(\mathcal{F}(0))$ and that

$$
\left\{\begin{array}{l}
\operatorname{div} u_{0}=0 \quad \text { in } \mathcal{F} \\
u_{0}=0 \quad \text { on } \partial \Omega \\
u_{0}=g_{0}+\omega_{0} x^{\perp}+w^{*}(x, 0) \quad \text { on } \partial \mathcal{S} \\
\operatorname{dist}(\mathcal{S}, \partial \Omega)>0
\end{array}\right.
$$

Then there exists $T_{\max }>0$ such that:

- For every $T \in\left(0, T_{\max }\right)$ the system (2.12)-(2.21) admits a unique strong solution on $[0, T]$.

- One of the following alternatives holds:

(i) $T_{\max }=\infty$;

(ii) $\lim _{t \rightarrow T_{\max }} \operatorname{dist}(\mathcal{S}(t), \partial \Omega)=0$.

\section{A local existence result}

In this section, we prove the local in time existence of strong solutions for the system (2.12)-(2.21). More precisely we show that the following result holds.

Theorem 4.1. Let $\varepsilon>0, C_{1}>0$. Assume that $X^{*}$ and $u_{0}$ satisfy the assumptions in Theorem 3.2, that $\left\|u_{0}\right\|_{\mathcal{H}^{1}(\mathcal{F})} \leqslant C_{1}$ and that $\operatorname{dist}(\mathcal{S}, \partial \Omega)>\varepsilon$. Then there exists $T_{0}>0$, depending only on $\varepsilon$ and $C_{1}$, such that, for every $T \in\left(0, T_{0}\right)$, there exists a unique strong solution of (2.12)-(2.21) on $[0, T]$.

Proof. The proof of this theorem is based on ideas and techniques quite similar to those in [31]. The main difference with respect to the proof in [31] consists in the construction of a change of variables transforming the Navier-Stokes equations in the non cylindrical domain $\mathcal{F}_{T}$ into a system of PDE's in the cylindrical domain $\mathcal{F} \times(0, T)$. For this reason, we only describe below the first step of the proof, consisting in the construction of this change of variables. The remaining part of the proof is similar to the proof of the corresponding result for rigid immersed bodies so that we refer to [31] for further details.

By a slight variation of the argument in Ladyzhenskaya [22, p.27] it can be shown that, for every $\delta>0$, there exists a continuous function $\left(x^{*}, t\right) \mapsto \Lambda^{*}\left(x^{*}, t\right)$ such that, for every $t \geqslant 0$, the map $x^{*} \mapsto \Lambda^{*}\left(x^{*}, t\right)$ is $C^{\infty}$ on $\overline{\mathcal{F}(t)}$ and such that, for every $x^{*} \in \mathcal{F}(t)$, the function $t \mapsto \Lambda^{*}\left(x^{*}, t\right)$ is of class $C^{\infty}$.

$$
\left\{\begin{aligned}
\operatorname{div} \Lambda^{*} & =0 & & \text { in } \mathbb{R}^{2} \backslash \overline{\mathcal{S}^{*}(t)}, t \in(0, T) \\
\Lambda^{*}\left(x^{*}, t\right) & =0 & & \text { if } \operatorname{dist}\left(x^{*}, \mathcal{S}^{*}(t)\right) \geqslant \delta>0, t \in(0, T), \\
\Lambda^{*}\left(x^{*}, t\right) & =w^{*}\left(x^{*}, t\right) & & \text { if } x^{*} \in \mathcal{S}^{*}(t), t \in(0, T)
\end{aligned}\right.
$$

We then define $\Lambda$ by

$$
\Lambda(x, t)=R_{\theta} \Lambda^{*}\left(R_{-\theta}(x-\xi(t)), t\right) \quad \forall x \in \mathbb{R}^{2}, \quad t \in(0, T) .
$$


Assume that there exists $T>0$ such that

$$
\operatorname{dist}(\mathcal{S}(t), \partial \Omega) \geqslant \delta \quad \forall t \in[0, T]
$$

Then the function $\Lambda$ satisfies

$$
\begin{gathered}
\operatorname{div} \Lambda=0 \text { in } \mathbb{R}^{2} \backslash \overline{\mathcal{S}(t)}, t \in(0, T), \\
\Lambda=0 \text { on } \partial \Omega, t \in(0, T), \\
\Lambda=w \text { in } \mathcal{S}(t), t \in(0, T) .
\end{gathered}
$$

From the regularity of $\Lambda^{*}$ mentioned above and the definition (4.2) of $\Lambda$ it follows that there exists a constant $C>0$ such that

$$
\left|\frac{\partial \Lambda}{\partial t}\left(x_{1}, x_{2}, t\right)\right|+\left|\frac{\partial^{k_{1}+k_{2}} \Lambda}{\partial x_{1}^{k_{1}} \partial x_{2}^{k_{2}}}\left(x_{1}, x_{2}, t\right)\right| \leqslant C\left(1+\left|\xi^{\prime}(t)\right|+\left|\theta^{\prime}(t)\right|\right),
$$

for all $t \in[0, T],\left(x_{1}, x_{2}\right) \in \mathcal{F}(t)$ and $k_{1}, k_{2} \in\{0,1,2\}$.

We next consider $z: \mathbb{R}^{2} \times[0, T] \rightarrow \mathbb{R}^{2}$ defined by

$$
z(x, t)=\left(\xi^{\prime}(t) \cdot x^{\perp}+\frac{\theta^{\prime}(t)}{2}|x-\xi(t)|^{2}\right) \operatorname{rot}(\tau)+\tau\left(\xi^{\prime}(t)+\theta^{\prime}(t)(x-\xi(t))^{\perp}\right)+\Lambda(x, t),
$$

where $\tau$ is smooth function with compact support in

$$
\Omega_{-\delta / 2}=\left\{x \in \Omega \quad \operatorname{dist}(x, \partial \Omega)>\frac{\delta}{2}\right\}
$$

and equal to 1 in $\Omega_{-\delta}$. For $t \geqslant 0$, the function $x \mapsto z(x, t)$ has a compact support and for $x \in \Omega_{-\delta}$ and $t \geqslant 0$, we have that

$$
z(x, t)=\xi^{\prime}(t)+\theta^{\prime}(x-\xi(t))^{\perp}+\Lambda(x, t) .
$$

This property implies, by using the relation $\Lambda(x, t)=w(x, t)$ for $x \in \mathcal{S}(t)$, that if $\mathcal{S}(t) \subset$ $\Omega_{-\delta}$, then, for all $y \in \mathcal{S}$, the function

$$
X(y, t)=\xi(t)+R_{\theta(t)} X^{*}(y, t)
$$

satisfies the initial value problem

$$
\frac{\mathrm{d} X}{\mathrm{~d} t}(y, t)=z(X(y, t), t), \quad X(y, 0)=y .
$$

Since the above initial value problem makes sense for every $y \in \mathbb{R}^{2}$ we still denote by $X$ the flow on $\mathbb{R}^{2}$ associated to (4.5). Thus, for every $t \in[0, T], X(\cdot, t)$ is a $C^{\infty}$-diffeomorphism from $\Omega$ onto $\Omega$ such that $X(\mathcal{F}, t)=\mathcal{F}(t)$ and $X(\mathcal{S}, t)=\mathcal{S}(t)$. The inverse of $X(\cdot, t)$ is denoted by $Y(\cdot, t)$ and the Jacobian matrix of $Y(\cdot, t)$ (respectively $X(\cdot, t)$ ) is denoted by $J_{Y}(\cdot, t)$ (respectively $J_{X}(\cdot, t)$ ).

By using the mapping $X$ constructed above, it is easy to see that the system (2.12)-(2.21) is equivalent to a system in the fixed domain $\mathcal{F} \times(0, T)$ of unknowns

$$
U(y, t)=J_{Y}(X(y, t), t) u(X(y, t), t), \quad P(y, t)=p(X(y, t), t) \quad(y \in \mathcal{F}, t \in(0, T)),
$$


and

$$
\Xi(t)=\int_{0}^{t} R_{-\theta(s)} \xi^{\prime}(s) \mathrm{d} s \quad(t \in[0, T]) .
$$

Notice that the vector field $U$ is of free divergence (see, for instance, Inoue and Wakimoto [19]). As we mentioned at the beginning of the proof, the local in time existence and uniqueness of solutions for the transformed system can be obtained by following closely the method on [31]. By using the inverse transformation $Y$ we get the local in time existence and uniqueness of solutions for (2.12)-(2.21).

\section{Proof of the main result}

This section is devoted to the proof of Theorem 3.2. We first prove an energy estimate.

Proposition 5.1. Let $(u, p, \xi, \theta)$ be a strong solution of $(2.12)-(2.21)$ defined on $[0, T)$, with $T>0$. Moreover, assume that there exists $\delta>0$ such that

$$
\operatorname{dist}(\mathcal{S}(t), \partial \Omega) \geqslant \delta \quad(t \in[0, T)) .
$$

Then there exists a constant $C_{T, \delta}>0$ such that,

$$
\begin{aligned}
\|u\|_{L^{\infty}\left(0, T ; \mathcal{L}^{2}(\mathcal{F}(t))\right.}+\|u\|_{L^{2}\left(0, T ; \mathcal{H}^{1}(\mathcal{F}(t))\right.}+ & \left\|\xi^{\prime}\right\|_{L^{\infty}\left(0, T ; \mathbb{R}^{2}\right)}+\left\|\theta^{\prime}\right\|_{L^{\infty}(0, T ; \mathbb{R})} \\
& \leqslant C_{T, \delta}\left(\left\|u_{0}\right\|_{L^{2}(\mathcal{F})}^{2}+\left|g_{0}\right|^{2}+\left|\omega_{0}\right|^{2}+1\right)
\end{aligned}
$$

Proof. Denote $v=u-\Lambda$, where $\Lambda$ is defined in (4.2). Then, for $t \in(0, T)$, we have

$$
\left\{\begin{array}{rlrl}
\frac{\partial v}{\partial t}+(u \cdot \nabla) v-\nu \Delta u+\nabla p & =-(v \cdot \nabla) \Lambda-(\Lambda \cdot \nabla) \Lambda-\frac{\partial \Lambda}{\partial t} & & \text { in } \mathcal{F}(t), \\
\operatorname{div} v & =0 & & \text { in } \mathcal{F}(t) \\
v & =0 & & \text { on } \partial \Omega, \\
v & =\xi^{\prime}(t)+\theta^{\prime}(t)(x-\xi(t))^{\perp} & & \text { on } \partial \mathcal{S}(t), \\
m \xi^{\prime \prime} & =-\int_{\partial \mathcal{S}(t)} \sigma(u, p) n \mathrm{~d} \Gamma & \\
\left(I \theta^{\prime}\right)^{\prime} & =-\int_{\partial \mathcal{S}(t)}(x-\xi)^{\perp} \cdot \sigma(u, p) n \mathrm{~d} \Gamma . &
\end{array}\right.
$$

If we take the inner product in $\mathbb{R}^{2}$ of the first equation by $v$ and we integrate the result on $\mathcal{F}(t)$ we obtain that

$$
\begin{aligned}
\int_{\mathcal{F}(t)}\left(\frac{\partial v}{\partial t}+[(u \cdot \nabla) v]\right) \cdot v \mathrm{~d} x-\int_{\mathcal{F}(t)} \operatorname{div} & \sigma(u, p) \cdot v \mathrm{~d} x=-\int_{\mathcal{F}(t)}[(v \cdot \nabla) \Lambda] \cdot v \mathrm{~d} x \\
& -\int_{\mathcal{F}(t)}[(\Lambda \cdot \nabla) \Lambda] \cdot v \mathrm{~d} x-\int_{\mathcal{F}(t)} \frac{\partial \Lambda}{\partial t} \cdot v \mathrm{~d} x .
\end{aligned}
$$

The above formula, summed up with the fifth equation of (5.3) multiplied by $\xi^{\prime}$ and the sixth one multiplied by $\theta^{\prime}$, combined to some integrations by parts and to the Reynolds 
transport formula gives:

$$
\begin{array}{rl}
\frac{1}{2} \frac{\mathrm{d}}{\mathrm{d} t} \int_{\mathcal{F}(t)}|v|^{2} \mathrm{~d} x+ & 2 \nu \int_{\mathcal{F}(t)}|D(v)|^{2} \mathrm{~d} x+\frac{1}{2} m \frac{\mathrm{d}}{\mathrm{d} t}\left|\xi^{\prime}\right|^{2}+\frac{\mathrm{d}}{\mathrm{d} t}\left(I \theta^{\prime}\right) \theta^{\prime} \\
=-\int_{\mathcal{F}(t)}[(v \cdot \nabla) \Lambda] \cdot v & \mathrm{~d} x-\int_{\mathcal{F}(t)}[(\Lambda \cdot \nabla) \Lambda] \cdot v \mathrm{~d} x \\
& -\int_{\mathcal{F}(t)} \frac{\partial \Lambda}{\partial t} \cdot v \mathrm{~d} x-2 \nu \int_{\mathcal{F}(t)} D(\Lambda): D(v) \mathrm{d} x .
\end{array}
$$

By using (4.3) it follows that there exists $C_{1}>0$ (depending only on $\delta$ ) such that

$$
\begin{aligned}
\frac{1}{2} \frac{\mathrm{d}}{\mathrm{d} t} \int_{\mathcal{F}(t)}|v|^{2} \mathrm{~d} x+2 \nu \int_{\mathcal{F}(t)}|D(v)|^{2} \mathrm{~d} x & +\frac{1}{2} m \frac{\mathrm{d}}{\mathrm{d} t}\left|\xi^{\prime}\right|^{2}+\frac{1}{2} \frac{\mathrm{d}}{\mathrm{d} t}\left(I\left|\theta^{\prime}\right|^{2}\right) \\
& \leqslant C_{1}\left(1+\int_{\mathcal{F}(t)}|v|^{2} \mathrm{~d} x+\left|\xi^{\prime}(t)\right|^{2}+\left|\theta^{\prime}(t)\right|^{2}\right) .
\end{aligned}
$$

By applying the Gronwall's lemma we obtain the desired conclusion.

We are now in a position to prove the main result.

Proof of Theorem 3.2. Let $T>0$ and let $(u, p, \xi, \theta)$ be a strong solution of (2.12)-(2.21). In order to prove the local solution obtained in Theorem 4.1 can be extended to a global one, it clearly suffices to show that if there exists $\delta>0$ such that dist $(\mathcal{S}(t), \partial \Omega) \geqslant \delta$ for all $t \in(0, T)$ then there exists a constant $C_{T}>0$ with,

$$
\|u(t)\|_{\mathcal{H}^{1}(\mathcal{F}(t))} \leqslant C_{T} \quad \forall t \in[0, T) .
$$

Recall that $v=u-\Lambda$ satisfies (5.3). By taking the inner product of the first equation in (5.3) with $\frac{\partial v}{\partial t}$ we obtain

$$
\begin{array}{r}
\left\|\frac{\partial v}{\partial t}\right\|_{\mathcal{L}^{2}(\mathcal{F}(t))}^{2}-\int_{\mathcal{F}(t)} \operatorname{div} \sigma(u, p) \cdot \frac{\partial v}{\partial t} \mathrm{~d} x=-\int_{\mathcal{F}(t)}[(u \cdot \nabla) v] \cdot \frac{\partial v}{\partial t} \mathrm{~d} x-\int_{\mathcal{F}(t)}[(v \cdot \nabla) \Lambda] \cdot \frac{\partial v}{\partial t} \mathrm{~d} x \\
-\int_{\mathcal{F}(t)}[(\Lambda \cdot \nabla) \Lambda] \cdot \frac{\partial v}{\partial t} \mathrm{~d} x-\int_{\mathcal{F}(t)} \frac{\partial \Lambda}{\partial t} \cdot \frac{\partial v}{\partial t} \mathrm{~d} x, \quad \text { a.e in }(0, T),
\end{array}
$$

and thus

$$
\begin{gathered}
\left\|\frac{\partial v}{\partial t}\right\|_{\mathcal{L}^{2}(\mathcal{F}(t))}^{2}-\int_{\mathcal{F}(t)} \operatorname{div} \sigma(v, p) \cdot \frac{\partial v}{\partial t} \mathrm{~d} x=-\int_{\mathcal{F}(t)}[(v \cdot \nabla) v] \cdot \frac{\partial v}{\partial t} \mathrm{~d} x-\int_{\mathcal{F}(t)}[(\Lambda \cdot \nabla) v] \cdot \frac{\partial v}{\partial t} \mathrm{~d} x \\
-\int_{\mathcal{F}(t)}[(v \cdot \nabla) \Lambda] \cdot \frac{\partial v}{\partial t} \mathrm{~d} x-\int_{\mathcal{F}(t)}[(\Lambda \cdot \nabla) \Lambda] \cdot \frac{\partial v}{\partial t} \mathrm{~d} x-\int_{\mathcal{F}(t)} \frac{\partial \Lambda}{\partial t} \cdot \frac{\partial v}{\partial t} \mathrm{~d} x \\
+\nu \int_{\mathcal{F}(t)} \Delta \Lambda \cdot \frac{\partial v}{\partial t} \mathrm{~d} x . \quad \text { a.e in }(0, T) .
\end{gathered}
$$

Using a density argument (see, for instance, [10], [9]), and the facts that

$$
v(x, t)=\xi^{\prime}(t)+\theta^{\prime}(t)(x-\xi(t))^{\perp} \quad \text { on } \partial \mathcal{S}(t), t \in(0, T)
$$


and

$$
v(x, t)=0 \quad \text { on } \partial \Omega, t \in(0, T)
$$

we obtain

$$
\begin{aligned}
& -\int_{\mathcal{F}(t)} \operatorname{div} \sigma(v, p) \cdot \frac{\partial v}{\partial t} \mathrm{~d} x=\nu \frac{\mathrm{d}}{\mathrm{d} t} \int_{\mathcal{F}(t)}|D(v)|^{2} \mathrm{~d} x+m\left|\xi^{\prime \prime}(t)\right|^{2}+I\left(\theta^{\prime \prime}\right)^{2} \\
& -m \theta^{\prime}\left(\xi^{\prime}\right)^{\perp} \cdot \xi^{\prime \prime}+I^{\prime} \theta^{\prime} \theta^{\prime \prime}+2 \nu \int_{\partial \mathcal{S}(t)} D(\Lambda) n \cdot\left(\xi^{\prime \prime}-\theta^{\prime}\left(\xi^{\prime}\right)^{\perp}+\theta^{\prime \prime}(x-\xi)^{\perp}\right) \mathrm{d} \Gamma .
\end{aligned}
$$

Combining (5.5) and (5.6) yields

$$
\begin{aligned}
\left\|\frac{\partial v}{\partial t}\right\|_{\mathcal{L}^{2}(\mathcal{F}(t))}^{2}+\nu \frac{\mathrm{d}}{\mathrm{d} t} \int_{\mathcal{F}(t)} \mid & |D(v)|^{2} \mathrm{~d} x+m\left|\xi^{\prime \prime}(t)\right|^{2}+I\left(\theta^{\prime \prime}\right)^{2} \\
& =\int_{\mathcal{F}(t)} \mathbb{F}_{1} \cdot \frac{\partial v}{\partial t} \mathrm{~d} x+\mathbb{F}_{2} \cdot \xi^{\prime \prime}+\mathbb{F}_{3} \theta^{\prime \prime}+\mathbb{F}_{4} \quad \text { a.e in }(0, T),
\end{aligned}
$$

with

$$
\begin{gathered}
\mathbb{F}_{1}=-([(v \cdot \nabla) v]+[(\Lambda \cdot \nabla) v]+[(v \cdot \nabla) \Lambda]+[(\Lambda \cdot \nabla) \Lambda])-\frac{\partial \Lambda}{\partial t}+\nu \Delta \Lambda \\
\mathbb{F}_{2}=m \theta^{\prime}\left(\xi^{\prime}\right)^{\perp}-2 \nu \int_{\partial \mathcal{S}(t)} D(\Lambda) n \mathrm{~d} \Gamma \\
\mathbb{F}_{3}=-I^{\prime} \theta^{\prime}-2 \nu \int_{\partial \mathcal{S}(t)} D(\Lambda) n \cdot(x-\xi)^{\perp} \mathrm{d} \Gamma \\
\mathbb{F}_{4}=2 \nu \int_{\partial \mathcal{S}(t)} D(\Lambda) n \cdot \theta^{\prime}\left(\xi^{\prime}\right)^{\perp} \mathrm{d} \Gamma
\end{gathered}
$$

From the Cauchy-Schwarz inequality, combined to the fact that $\xi^{\prime}$ and $\theta^{\prime}$ are bounded in $[0, T)$ (by Proposition 5.1), we obtain that for a.e. $t \in(0, T)$ we have

$$
\begin{aligned}
\frac{1}{2}\left\|\frac{\partial v}{\partial t}\right\|_{\mathcal{L}^{2}(\mathcal{F}(t))}^{2}+\nu \frac{\mathrm{d}}{\mathrm{d} t} \int_{\mathcal{F}(t)}|D(v)|^{2} \mathrm{~d} x+\frac{m}{2}\left|\xi^{\prime \prime}(t)\right|^{2} & +\frac{I}{2}\left|\theta^{\prime \prime}(t)\right|^{2} \\
& \leqslant C_{0}\left(1+\|(v \cdot \nabla) v\|_{\mathcal{L}^{2}(\mathcal{F}(t))}^{2}\right)
\end{aligned}
$$

where $C_{0}$ is a positive constant which depends on $\delta,\left\|u_{0}\right\|_{\mathcal{H}^{1}(\mathcal{F})}, g_{0}$ and $\omega_{0}$.

By applying a Hölder's inequality, combined to the continuous embedding of $H^{1 / 2}(\mathcal{F}(t))$ in $L^{4}(\mathcal{F}(t))$ and to an interpolation's inequality (see, for instance, Lions and Magenes [25, pp. 23]), we get that there exists a constant $C_{1}>0$ such that for a.e. $t \in(0, T)$,

$$
\begin{aligned}
\int_{\mathcal{F}(t)}|(v \cdot \nabla) v|^{2} \mathrm{~d} x & \leqslant\|v\|_{\mathcal{L}^{4}(\mathcal{F}(t))}^{2}\|\nabla v\|_{\left[L^{4}(\mathcal{F}(t))\right]^{4}}^{2} \\
& \leqslant C_{1}\|v\|_{\mathcal{L}^{2}(\mathcal{F}(t))}\|v\|_{\mathcal{H}^{1}(\mathcal{F}(t))}\|\nabla v\|_{\left[L^{2}(\mathcal{F}(t))\right]^{4}}\|\nabla v\|_{\left[H^{1}(\mathcal{F}(t))\right]^{4}} \\
\leqslant & C_{1}\|v\|_{\mathcal{L}^{2}(\mathcal{F}(t))}\|v\|_{\mathcal{H}^{1}(\mathcal{F}(t))}^{2}\left\{\|\nabla v\|_{\left[L^{2}(\mathcal{F}(t))\right]^{4}}+\sum_{i=1}^{2}\left\|D^{2} v_{i}\right\|_{\left[L^{2}(\mathcal{F}(t))\right]^{4}}\right\}
\end{aligned}
$$


On the other hand, we can consider $(v, p)$ as the solution of a stationary Stokes problem, depending on the parameter $t>0$,

$$
\begin{aligned}
-\nu \Delta v+\nabla p=f & \text { in } \mathcal{F}(t), \\
\operatorname{div} v=0 & \text { in } \mathcal{F}(t), \\
v=\xi^{\prime}(t)+\theta^{\prime}(t)[x-\xi(t)]^{\perp} & \text { on } \partial \mathcal{S}(t), \\
v=0, & \text { on } \partial \Omega,
\end{aligned}
$$

where

$$
f=-\frac{\partial v}{\partial t}+\mathbb{F}_{1}
$$

By using the classical elliptic regularity of the Stokes system, we obtain that there exists a constant $C_{2}=C_{2}(\nu, \Omega, \mathcal{S})>0$ such that

$$
\|\nabla v\|_{\left[L^{2}(\mathcal{F}(t))\right]^{4}}+\sum_{i=1}^{2}\left\|D^{2} v_{i}\right\|_{\left[L^{2}(\mathcal{F}(t))\right]^{4}} \leqslant C_{2}\left(\|f\|_{\mathcal{L}^{2}(\mathcal{F}(t))}+\left|\xi^{\prime}\right|+\left|\theta^{\prime}\right|\right) .
$$

(Here the constant $C_{2}$ does not depend on $t$ since the assumption (5.1) holds true.)

From (5.9) and (5.10) together with the above inequality, we deduce that

$$
\begin{aligned}
\|(v \cdot \nabla) v\|_{\mathcal{L}^{2}(\mathcal{F}(t))}^{2} \leqslant C_{3}\|v\|_{\mathcal{L}^{2}(\mathcal{F}(t))}\|v\|_{\mathcal{H}^{1}(\mathcal{F}(t))}^{2}\left(\left\|\frac{\partial v}{\partial t}\right\|_{\mathcal{L}^{2}(\mathcal{F}(t))}\right. & +\|(v \cdot \nabla) v\|_{\mathcal{L}^{2}(\mathcal{F}(t))} \\
& \left.+\|v\|_{\mathcal{H}^{1}(\mathcal{F}(t))}^{2}+1+\left|\xi^{\prime}\right|^{2}+\left|\theta^{\prime}\right|^{2}\right) .
\end{aligned}
$$

Using the energy estimate (5.2) of Proposition 5.1, we obtain that, for every $\varepsilon>0$ there exists $c_{\varepsilon}>0$ such that

$$
\|(v \cdot \nabla) v\|_{\mathcal{L}^{2}(\mathcal{F}(t))}^{2} \leqslant \varepsilon\left\|\frac{\partial v}{\partial t}\right\|_{\mathcal{L}^{2}(\mathcal{F}(t))}^{2}+c_{\varepsilon}\left(\|v\|_{\mathcal{H}^{1}(\mathcal{F}(t))}^{4}+\|v\|_{\mathcal{H}^{1}(\mathcal{F}(t))}^{2}\right)
$$

Combining the above inequality (for $\varepsilon$ small enough) with (5.8) yields

$$
\begin{aligned}
\frac{1}{4}\left\|\frac{\partial v}{\partial t}\right\|_{\mathcal{L}^{2}(\mathcal{F}(t))}^{2}+\nu \frac{\mathrm{d}}{\mathrm{d} t} \int_{\mathcal{F}(t)}|D(v)|^{2} \mathrm{~d} x+\frac{m}{2}\left|\xi^{\prime \prime}(t)\right|^{2}+\frac{I}{2}\left|\theta^{\prime \prime}(t)\right|^{2} & \\
& \leqslant C_{4}\left(1+\|v\|_{\mathcal{H}^{1}(\mathcal{F}(t))}^{4}\right)
\end{aligned}
$$

for a.e. $t \in(0, T)$. The positive constant $C_{4}$ depends on $\delta,\left\|u_{0}\right\|_{\mathcal{H}^{1}(\mathcal{F})}, g_{0}$ and $\omega_{0}$.

If we extend $v$ to the whole domain $\Omega$ by setting

$$
v(x, t)=\xi^{\prime}(t)+\theta^{\prime}(t)[x-\xi(t)]^{\perp} \quad \forall x \in \mathcal{S}(t), t \in(0, T),
$$

then we have that $v(t) \in \mathcal{H}_{0}^{1}(\Omega)$ and, by using the Poincaré inequality we obtain

$$
\|v\|_{\mathcal{H}^{1}(\mathcal{F}(t))} \leqslant\|v\|_{\mathcal{H}^{1}(\Omega)} \leqslant C_{\Omega}\|\nabla v\|_{\left[L^{2}(\Omega)\right]^{4}}
$$


The above estimate, combined to the facts that $\operatorname{div} v=0$ in $\Omega$ and that $D(v)=0$ in $\mathcal{S}(t)$, implies that

$$
\|v\|_{\mathcal{H}^{1}(\mathcal{F}(t))} \leqslant C\left(\int_{\mathcal{F}(t)}|D(v)|^{2} \mathrm{~d} x\right)^{\frac{1}{2}} .
$$

Combining the above relation with (5.12), (4.3) and Proposition 5.1, we get after applying the Gronwall inequality, that

$$
\left\|\frac{\partial v}{\partial t}\right\|_{\mathcal{L}^{2}\left(\mathcal{F}_{T}\right)}+\left\|\xi^{\prime \prime}\right\|_{L^{2}\left(0, T ; \mathbb{R}^{2}\right)}+\left\|\theta^{\prime \prime}\right\|_{L^{2}(0, T ; \mathbb{R})}+\|v\|_{L^{\infty}\left(0, T ; \mathcal{H}^{1}(\Omega)\right)} \leqslant C_{T} .
$$

This concludes the proof of Theorem 3.2.

\section{A mixed formulation and a time discretization method}

In this section, based on a weak form of the governing equations, we describe a method for the time discretization of (2.12)-(2.21). This method is a version of the "global method" introduced in San Martín, Scheid, Takahashi and Tucsnak [28] for the numerical analysis of the motion of rigid bodies in a viscous fluid.

We first define some function spaces. Let $(\xi(t), \theta(t))_{t \geqslant 0}$ be a trajectory of the solid such that $\mathcal{S}(\xi(t), \theta(t), t) \subset \Omega$ for every $t \geqslant 0$. We denote, for every $t \geqslant 0$,

$$
\begin{gathered}
\mathcal{K}(\xi(t), \theta(t), t)=\left\{u \in \mathcal{H}_{0}^{1}(\Omega) \mid D(u)=0 \quad \text { in } \quad \mathcal{S}(\xi(t), \theta(t), t)\right\}, \\
\mathcal{M}(\xi(t), \theta(t), t)=\left\{p \in L^{2}(\Omega) \mid \int_{\Omega} p \mathrm{~d} x=0 \quad \text { and } p=0 \text { in } \quad \mathcal{S}(\xi(t), \theta(t), t)\right\},
\end{gathered}
$$

where $D(u)$ is the strain rate tensor defined by (2.18).

According to Lemma 1.1 of [33, pp.18], for any $u \in \mathcal{K}(\xi(t), \theta(t), t)$, there exist $\ell_{u} \in \mathbb{R}^{2}$ and $\omega_{u} \in \mathbb{R}$ such that

$$
u(x)=\ell_{u}+\omega_{u}(x-\xi)^{\perp} \quad \forall x \in \mathcal{S}(\xi(t), \theta(t), t) .
$$

Let $(u, p, \xi, \theta)$ be a strong solution of (2.12)-(2.21). The vector velocity field $u$ and the pressure $p$ can be extended to $\Omega$ by setting

$$
\begin{gathered}
u(x, t)=\xi^{\prime}(t)+\theta^{\prime}(t)(x-\xi(t))^{\perp}+w(x, t) \quad \text { if } \quad x \in \mathcal{S}(\xi(t), \theta(t), t), \\
p(x, t)=0 \quad \text { if } \quad x \in \mathcal{S}(\xi(t), \theta(t), t) .
\end{gathered}
$$

The extended vector $u(t)$ belongs to $\mathcal{H}_{0}^{1}(\Omega)$. In the remaining part of this paper, the solution $u$ and $p$ of (2.12)-(2.21) will be extended as above.

We also need to extend the density field $\rho$ of the creature (defined in (2.9)) to the whole domain $\Omega$ by setting

$$
\rho(x, t)=1 \text { for all } x \in \mathcal{F}(\xi(t), \theta(t), t), \quad t \geqslant 0 .
$$

Let $\varphi: \bar{\Omega} \rightarrow \mathbb{R}$ be a smooth scalar field and $v: \bar{\Omega} \rightarrow \mathbb{R}$ be a smooth vector field. The material derivative of $\varphi$ is defined by

$$
\frac{\mathrm{d} \varphi}{\mathrm{d} t}=\frac{\partial \varphi}{\partial t}+u \cdot \nabla \varphi
$$


whereas the material derivative of $v$ writes as

$$
\frac{\mathrm{d} v}{\mathrm{~d} t}=\frac{\partial v}{\partial t}+(u \cdot \nabla) v
$$

It is well known that for every smooth scalar fields $\varphi$ and $\psi$, we have

$$
\begin{gathered}
\frac{\mathrm{d}(\varphi \psi)}{\mathrm{d} t}=\frac{\mathrm{d} \varphi}{\mathrm{d} t} \psi+\varphi \frac{\mathrm{d} \psi}{\mathrm{d} t}, \\
\frac{\mathrm{d}}{\mathrm{d} t} \int_{\mathcal{S}(\xi, \theta, t)} \rho \varphi \mathrm{d} x=\int_{\mathcal{S}(\xi, \theta, t)} \rho \frac{\mathrm{d} \varphi}{\mathrm{d} t} \mathrm{~d} x .
\end{gathered}
$$

In order to give the global weak formulation of our problem, we need the bilinear forms

$$
a: \mathcal{H}_{0}^{1}(\Omega) \times \mathcal{H}_{0}^{1}(\Omega) \rightarrow \mathbb{R}, \quad b: \mathcal{H}_{0}^{1}(\Omega) \times L^{2}(\Omega) \rightarrow \mathbb{R},
$$

defined by

$$
\begin{gathered}
a(u, v)=2 \nu \int_{\Omega} D(u): D(v) \mathrm{d} x \quad \forall u, v \in \mathcal{H}_{0}^{1}(\Omega), \\
b(u, q)=-\int_{\Omega}(\operatorname{div} u) q \mathrm{~d} x \quad \forall u \in \mathcal{H}_{0}^{1}(\Omega), \quad q \in L^{2}(\Omega) .
\end{gathered}
$$

Proposition 6.1. Let $(u, p, \xi, \theta)$ be a strong solution of (2.12)-(2.21) where $u$ and $p$ are extended to $\Omega$ as above. Then, we have

$$
\begin{gathered}
\int_{\Omega} \rho \frac{\mathrm{d} u}{\mathrm{~d} t} \cdot v \mathrm{~d} x+a(u, v)+b(v, p)=0 \quad \forall v \in \mathcal{K}(\xi, \theta, t), \\
b(u, q)=0 \quad \forall q \in \mathcal{M}(\xi, \theta, t)
\end{gathered}
$$

for a.e. $t \in(0, T)$.

Conversely, assume that $(u, p, \xi, \theta)$ has the properties in (3.3) and that $u$ and $p$ satisfy (6.3)-(6.4) together with (6.8)-(6.9). Then $(u, p, \xi, \theta)$ is a strong solution of $(2.12)-(2.21)$.

Proof. In this proof we use the notation, introduced in Section 3

$$
\mathcal{S}(t)=\mathcal{S}(\xi(t), \theta(t), t), \quad \mathcal{F}(t)=\mathcal{F}(\xi(t), \theta(t), t) .
$$

Let $t \in[0, T]$. Equation (2.12) can be written as

$$
\rho \frac{\mathrm{d} u}{\mathrm{~d} t}-\operatorname{div} \sigma(u, p)=0 \quad \text { for } \quad x \in \mathcal{F}(t)
$$

By taking the inner product in $\mathbb{R}^{2}$ of the above equation by $v \in \mathcal{K}(\xi(t), \theta(t), t)$ and by integrating by parts on $\mathcal{F}(t)$ we obtain that

$$
\int_{\mathcal{F}(t)} \rho \frac{\mathrm{d} u}{\mathrm{~d} t} \cdot v \mathrm{~d} x+2 \nu \int_{\mathcal{F}(t)} D(u): D(v) \mathrm{d} x-\int_{\mathcal{F}(t)}(\operatorname{div} v) p \mathrm{~d} x-\int_{\partial \mathcal{S}(t)} \sigma(u, p) n \cdot v \mathrm{~d} \Gamma=0
$$

Since $D(v)=0$ in $\mathcal{S}(t)$, there exist $\ell \in \mathbb{R}^{2}$ and $\omega \in \mathbb{R}$ such that

$$
v(x)=\ell+\omega(x-\xi(t))^{\perp} \quad \forall x \in \mathcal{S}(t),
$$


so that,

$$
\int_{\partial \mathcal{S}(t)} \sigma(u, p) n \cdot v \mathrm{~d} \Gamma=\ell \cdot \int_{\partial \mathcal{S}(t)} \sigma(u, p) n \mathrm{~d} \Gamma+\omega \int_{\partial \mathcal{S}(t)}(x-\xi)^{\perp} \cdot \sigma(u, p) n \mathrm{~d} \Gamma .
$$

The above equation, combined to (2.16)-(2.17), yields that

$$
-\int_{\partial \mathcal{S}(t)} \sigma(u, p) n \cdot v \mathrm{~d} \Gamma=m \xi^{\prime \prime} \cdot \ell+\omega\left(I \theta^{\prime}\right)^{\prime} .
$$

The above formula and (6.10) imply that

$$
\int_{\mathcal{F}(t)} \rho \frac{\mathrm{d} u}{\mathrm{~d} t} \cdot v \mathrm{~d} x+2 \nu \int_{\mathcal{F}(t)} D(u): D(v) \mathrm{d} x-\int_{\mathcal{F}(t)} p \operatorname{div} v \mathrm{~d} x+m \xi^{\prime \prime} \cdot \ell+\omega\left(I \theta^{\prime}\right)^{\prime}=0
$$

On the other hand, the assumptions (H3) and (H4) imply that

$$
\int_{\mathcal{S}(t)} \rho w \mathrm{~d} x=0, \quad \int_{\mathcal{S}(t)} \rho(x-\xi)^{\perp} \cdot w \mathrm{~d} x=0
$$

so that,

$$
\int_{\mathcal{S}(t)} \rho u \mathrm{~d} x=m \xi^{\prime}(t), \quad \int_{\mathcal{S}(t)} \rho(x-\xi)^{\perp} \cdot u \mathrm{~d} x=I(t) \theta^{\prime}(t) .
$$

Since the above equations hold for every $t \in[0, T]$ we can take their derivatives with respect to $t$ to get

$$
\int_{\mathcal{S}(t)} \rho \frac{\mathrm{d} u}{\mathrm{~d} t} \mathrm{~d} x=m \xi^{\prime \prime}(t), \quad \int_{\mathcal{S}(t)} \rho(x-\xi)^{\perp} \cdot \frac{\mathrm{d} u}{\mathrm{~d} t} \mathrm{~d} x=\left(I \theta^{\prime}\right)^{\prime}(t)
$$

The above relation, combined to (6.11), implies that

$$
m \xi^{\prime \prime}(t) \cdot \ell+\omega\left(I \theta^{\prime}\right)^{\prime}(t)=\int_{\mathcal{S}(t)} \rho \frac{\mathrm{d} u}{\mathrm{~d} t} \cdot v \mathrm{~d} x .
$$

The above equation and (6.12) give the first implication.

For the converse, we notice that, since $(u, p, \xi, \theta)$ has the regularity property (3.3), we can integrate by parts in (6.8) to get that, for any $v \in \mathcal{K}(\mathcal{S}(t))$, we have

$$
\int_{\Omega} \rho \frac{\mathrm{d} u}{\mathrm{~d} t} \cdot v \mathrm{~d} x-\int_{\mathcal{F}(t)}(\operatorname{div} \sigma(u, p)) \cdot v \mathrm{~d} x+\int_{\partial \mathcal{S}(t)} \sigma(u, p) n \cdot v \mathrm{~d} \Gamma=0 .
$$

In particular, the above equation holds for any smooth function $v$ with compact support in $\mathcal{F}(t)$, so that $(u, p)$ satisfies $(2.12)$. Using this fact in (6.13), we get that

$$
\int_{\mathcal{S}(t)} \rho \frac{\mathrm{d} u}{\mathrm{~d} t} \cdot v \mathrm{~d} x=-\int_{\partial \mathcal{S}(t)} \sigma(u, p) n \cdot v \mathrm{~d} \Gamma
$$

Now using test functions such that $v=e_{i},(i=1,2)$ in $\mathcal{S}(t)$, we obtain

$$
\int_{\mathcal{S}(t)} \rho \frac{\mathrm{d} u}{\mathrm{~d} t} \mathrm{~d} x=-\int_{\partial \mathcal{S}(t)} \sigma(u, p) n \mathrm{~d} \Gamma \quad \forall v \in \mathcal{K}(\mathcal{S}(t))
$$

which yields, by using (6.7), equation (2.16). Finally, by using a test function such that $v=(x-\xi)^{\perp}$, in $\mathcal{S}(t)$, we obtain that

$$
\int_{\mathcal{S}(t)} \rho(x-\xi(t))^{\perp} \cdot \frac{\mathrm{d} u}{\mathrm{~d} t} \mathrm{~d} x=-\int_{\partial \mathcal{S}(t)}(x-\xi(t))^{\perp} \cdot \sigma(u, p) n \mathrm{~d} \Gamma,
$$

which, combined to (6.6) and (6.7), implies (2.17). 
By using the weak form derived above, we give below a discretization of the governing equations with respect to the time variable. Let us first divide the time interval $[0, T]$ into subintervals $\left[t^{k}, t^{k+1}\right]$ with $t^{k+1}-t^{k}=\Delta t$ and $k \in \mathbb{N} \cap\left[0, \frac{T}{\Delta t}\right]$. Let $\left(u^{k}, p^{k}, \xi^{k}, \theta^{k}\right)$ be the approximation of the solution of (6.8), (6.9) at time $t=t^{k}$ (remark that $u^{k}$ and $p^{k}$ are functions defined on all $\Omega$ ). We denote

$$
\begin{gathered}
\mathcal{K}^{k}=\mathcal{K}\left(\xi^{k}, \theta^{k}, t^{k}\right), \quad \mathcal{M}^{k}=\mathcal{M}\left(\xi^{k}, \theta^{k}, t^{k}\right), \\
\mathcal{S}^{k}=\mathcal{S}\left(\xi^{k}, \theta^{k}, t^{k}\right), \quad \mathcal{F}^{k}=\mathcal{F}\left(\xi^{k}, \theta^{k}, t^{k}\right),
\end{gathered}
$$

and we consider the functions

$$
\begin{array}{cr}
\Lambda^{k}(x)=R_{\theta^{k}} \Lambda^{*}\left(R_{-\theta^{k}}\left(x-\xi^{k}\right), t^{k}\right) & \forall x \in \mathbb{R}^{2}, \\
\rho^{k}(x)=\left\{\begin{array}{ll}
\rho^{*}\left(R_{-\theta^{k}}\left(x-\xi^{k}\right), t^{k}\right) & \text { if } x \in \mathcal{S}^{k} \\
1 & \text { if } x \in \mathcal{F}^{k}
\end{array} .\right.
\end{array}
$$

Now, let us describe the numerical procedure for approximating the solutions of (2.12)(2.21). This procedure is based on the weak form derived in Proposition 6.1.

The first step of our scheme consists in computing the new position of the mass center and the new orientation of the creature by setting

$$
\xi^{k+1}=\xi^{k}+\Delta t u^{k}\left(\xi^{k}\right), \quad \theta^{k+1}=\theta^{k}+\frac{\Delta t}{I\left(t_{k}\right)} \int_{\mathcal{S}^{k}} \rho^{k}\left(u^{k}(x)-u^{k}\left(\xi^{k}\right)\right) \cdot\left(x-\xi^{k}\right)^{\perp} \mathrm{d} x .
$$

The second step consists in computing the global velocity field $u^{k+1}$ and the global pressure field $p^{k+1}$. To this end, we use a high-order characteristics method to approximate the material derivative (see Boukir, Maday, Métivet and Razafindrakoto [2]). More precisely, we look for $u^{k+1} \in \Lambda^{k+1}+\mathcal{K}^{k+1}$ and $p^{k+1} \in \mathcal{M}^{k+1}$ such that, for all $\phi \in \mathcal{K}^{k+1}$ and for all $q \in \mathcal{M}^{k+1}$, we have

$$
\begin{gathered}
\frac{1}{2 \Delta t}\left(\rho^{k+1}\left(3 u^{k+1}-4 u^{k} \circ \bar{X}^{k}+u^{k-1} \circ \overline{\bar{X}}^{k-1}\right), \phi\right)+a\left(u^{k+1}, \phi\right)+b\left(\phi, p^{k+1}\right)=0 \\
b\left(u^{k+1}, q\right)=0 .
\end{gathered}
$$

In the above equations, the approximate characteristics are given by $\bar{X}^{k}(x)=\chi^{k}\left(t^{k} ; t^{k+1}, x\right)$ and $\overline{\bar{X}}^{k-1}(x)=\chi^{k}\left(t^{k-1} ; t^{k+1}, x\right)$ for all $x \in \Omega$, where $\chi^{k}$ is the solution of the problem

$$
\left\{\begin{aligned}
\frac{\mathrm{d}}{\mathrm{d} t} \chi^{k}\left(t ; t^{k+1}, x\right) & =u^{k *}\left(\chi^{k}\left(t ; t^{k+1}, x\right)\right), \quad t \in\left[t_{k-1}, t_{k+1}\right] \\
\chi^{k}\left(t^{k+1} ; t^{k+1}, x\right) & =x
\end{aligned}\right.
$$

with $u^{k *}=2 u^{k}-u^{k-1}$.

It is easy to see that, for any $k \in \mathbb{N}^{*} \cap\left[0, \frac{T}{\Delta t}\right]$, equations (6.15)-(6.16) represent a mixed formulation of a well-posed Stokes type system, so that our scheme is well defined. 


\section{Numerical simulations}

In this section we apply the time discretization method proposed above to several examples. For simulation purposes, the time discretization is coupled to a discretization with respect to the space variable. More precisely, we use a mixed finite-element method to solve (at each time step) (6.15)-(6.16). This discretization with respect to the space variable is similar to the method in [28], so we do not describe it here. The stability and convergence properties of the method will be analyzed in a forthcoming paper.

Consider a reference domain $\mathcal{S}_{0}$ which is assumed to be symmetric with respect to the horizontal axis. More precisely, $\mathcal{S}_{0}$ is defined by

$$
\mathcal{S}_{0}=\left\{\left(y_{1}, y_{2}\right) \in[0, l] \times \mathbb{R}, y_{2} \in\left[-\gamma\left(y_{1}\right), \gamma\left(y_{1}\right)\right]\right\}
$$

where $\gamma \in C^{\infty}(0, l) \cap C([0, l])$ is a given non-negative function with $\gamma(0)=\gamma(l)=0$ and $\gamma^{\prime}(0)=\infty$ and $\gamma^{\prime}(l)=-\infty$ (the last two conditions are necessary in order to have the smoothness of the domain field by the fluid). The imposed deformation of the fish-like swimmer can be chosen in various ways depending, in particular, on the organism we are trying to imitate (see, for instance, [4] or [26]). In this section we choose a deformation law inspired by Leroyer and Visonneau [23], which imitates eel-like swimming. According to [23], the deformation of the body is given by a beam model. This means that, during the deformation, the cross sections remain orthogonal to the middle axis (Kirchhoff model, see Fig. 1).

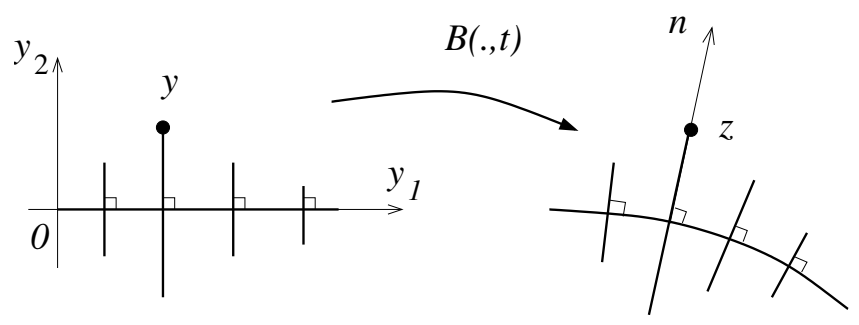

Figure 1: The beam model for the deformation $B$.

In order to define the deformation $X^{*}$ we consider, for every $t \geqslant 0$ a mapping

$$
\begin{aligned}
B(\cdot, t): \mathcal{S}_{0} & \rightarrow \mathbb{R}^{2} \\
y=\left(y_{1}, y_{2}\right) & \mapsto B(y, t)=f\left(y_{1}, t\right)+y_{2} n\left(y_{1}, t\right) .
\end{aligned}
$$

In the above definition, $f(\cdot, t)=\left(\begin{array}{l}f_{1}(\cdot, t) \\ f_{2}(\cdot, t)\end{array}\right) \in C^{\infty}\left([0, l], \mathbb{R}^{2}\right)$ is the parametrization of the middle line by its arc-coordinate $y_{1}$ (i.e. with $\left|\partial f / \partial y_{1}\right| \equiv 1$ ) at instant $t$ and $n$ is the normal unit vector. It is easy to see that, for every $t \geqslant 0$, the mapping $y \mapsto B(y, t)$ is in $C^{\infty}\left(\mathcal{S}_{0}, \mathbb{R}^{2}\right)$ and its jacobian is equal to

$$
\operatorname{det}(\nabla B)(y, t)=1-y_{2} \kappa\left(y_{1}, t\right) \text { for all } t \geqslant 0, \quad y \in \mathcal{S}_{0},
$$

where $\kappa\left(y_{1}, t\right)$ is the curvature of the middle line at the point of abscissa $y_{1}$, at time $t$.

In the remaining part of this section we assume that, for every $t \in(0, T)$, we have

$$
\inf _{y_{1} \in(0, l)} \frac{\partial f_{1}}{\partial y_{1}}\left(y_{1}, t\right)>0, \quad \sup _{y_{1} \in(0, l)}\left|\kappa\left(y_{1}, t\right) \gamma\left(y_{1}\right)\right|<1 .
$$


With the above assumptions, it is not difficult to prove that $B(\cdot, t)$ is a $C^{\infty}$-diffeomorphism from $\mathcal{S}_{0}$ onto $B\left(\mathcal{S}_{0}, t\right)$, for all $t \geqslant 0$. Therefore, assumption (H1) is satisfied with $X^{*}=B$. Due to the symmetry of $\mathcal{S}_{0}$, the beam model preserves the total area of the body, i.e., $X^{*}=B$ also satisfies assumption (H2).

The mapping $B$ does not preserve the linear and the angular momentum of the creature (i.e., the assumptions (H3) and (H4) do not hold with $X^{*}=B$ ). However, one can check that there exist two smooth functions $\theta_{B}:[0, T] \rightarrow \mathbb{R}$ and $\xi_{B}:[0, T] \rightarrow \mathbb{R}^{2}$ such that $X^{*}$ defined by

$$
X^{*}(y, t)=R_{\theta_{B}(t)}\left(B(y, t)-\xi_{B}(t)\right),
$$

satisfies the assumptions (H3) and (H4). Since assumptions (H1) and (H2) are invariant with respect to rotations and translations, we have that $X^{*}$ satisfies $(\mathrm{H} 1)-(\mathrm{H} 4)$.

An example of mapping $f$ to be used in (7.2) for the construction of $B$ is inspired by zoological observations (see [23]) and it can be constructed by first specifying the time variation of the curvature. More precisely, we set

$$
\kappa\left(y_{1}, t\right)=\left(a_{2} y_{1}^{2}+a_{1} y_{1}+a_{0}\right) \sin \left(2 \pi\left(y_{1} / \lambda-t / T\right)\right)+a_{3} \quad\left(y_{1} \in(0, l)\right),
$$

where $a_{i}, i \in\{1,2,3\}, \lambda, T$ are given constants. The mid-line $f=\left(f_{1}, f_{2}\right)$ is computed by integrating the Frenet formulae

$$
\frac{\partial^{2} f}{\partial y_{1}^{2}}\left(y_{1}, t\right)=\kappa\left(y_{1}, t\right)\left[\frac{\partial f}{\partial y_{1}}\left(y_{1}, t\right)\right]^{\perp} .
$$

If we choose $f(0, t)=(0,0)^{T}, \frac{\partial f}{\partial y_{1}}(0, t)=(1,0)^{T}$ for all $t \geqslant 0$ and we integrate $(7.7)$ with respect to $y_{1}$, we get for all $t \geqslant 0$,

$$
f\left(y_{1}, t\right)=\left(\int_{0}^{y_{1}} \cos (\alpha(z, t)) \mathrm{d} z,-\int_{0}^{y_{1}} \sin (\alpha(z, t)) \mathrm{d} z\right)^{T}
$$

with $\alpha\left(y_{1}, t\right)=-\int_{0}^{y_{1}} \kappa(z, t) \mathrm{d} z$. Notice that the above formula implies that if

$$
\frac{l^{3}\left|a_{2}\right|}{3}+\frac{l^{2}\left|a_{1}\right|}{2}+l\left(\left|a_{0}\right|+\left|a_{3}\right|\right)<\frac{\pi}{2},
$$

then $\inf _{y_{1} \in(0, l)} \frac{\partial f_{1}}{\partial y_{1}}\left(y_{1}, t\right)>0$ so that the first condition in (7.4) is satisfied. Moreover, if we choose $\gamma$ such that

$$
\sup _{y_{1} \in(0, l)}\left|\gamma\left(y_{1}\right)\right|<\frac{1}{l^{2}\left|a_{2}\right|+l\left|a_{1}\right|+\left|a_{0}\right|+\left|a_{3}\right|},
$$

then the second condition in (7.4) is also satisfied. Therefore, with the above choice of $f$, the map $B$ in (7.2) is indeed a $C^{\infty}$ diffeomorphism.

With the above ingredients for the deformation law, we performed three numerical tests. In each one of the tests below the Reynolds number is of order of $10^{4}$, which corresponds to the swimming regime of usual fishes (see, for instance, [6]).

Example 7.1. Straight line swimming.

In this example we choose, following [23], $a_{0}=1, a_{1}=0.5, a_{2}=2$ and $a_{3}=0, \lambda=1$ and $T=1, l=0.3$ in (7.6). In this case the periodic (with respect to time) deformation 


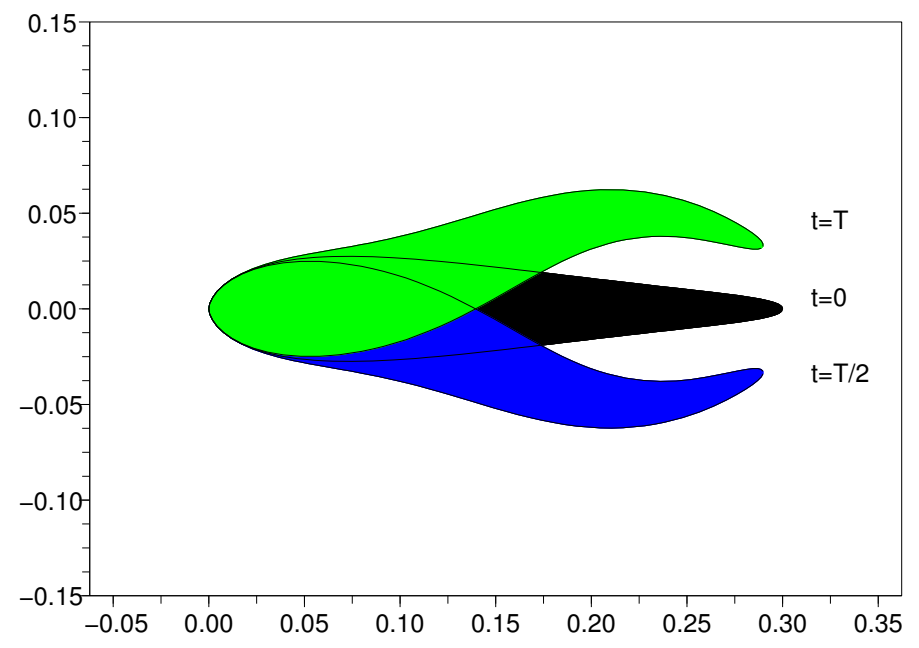

Figure 2: Deformation of the creature for the straight line swimming.

of the creature is symmetric with respect to the configuration in which the midline is a straight line (see Figure 2). As expected, the motion of the mass center of the creature is essentially a translation (see Figure 3). We refer to [4] and [26] for similar simulations, obtained with different numerical methods.

Example 7.2. Turning.

In this example we choose $a_{0}=0, a_{1}=0.5, a_{2}=3, a_{3}=-3$ and $\lambda=1, T=1, l=0.3$ in (7.6). In this case the time periodic deformation of the creature is symmetric with respect to its position in which the mid line has a constant curvature equal to $a_{3}$ (see Figure 4). This turning law differs from that in [23] by the fact that the creature is simultaneously advancing and turning. As expected the mass center of the creature describes a curved trajectory turning downwards (see Figure 5).

Example 7.3. Meeting.

This example takes advantage of one of the new features of our numerical method which is that we can tackle several swimmers in a bounded fluid. We consider two identic creatures animated by forward laws similar to that in Example 7.1 and oriented in opposite directions. We can notice in Figure 6 that the two creatures get very close, without touching, and that they continue their ways in opposite directions. This simulation can be seen as a new version of the "kiss and go" effect studied numerically, in the case of rigid bodies moving in a fluid, by Glowinski and Maury in [27]. 
$t=0.013 s$

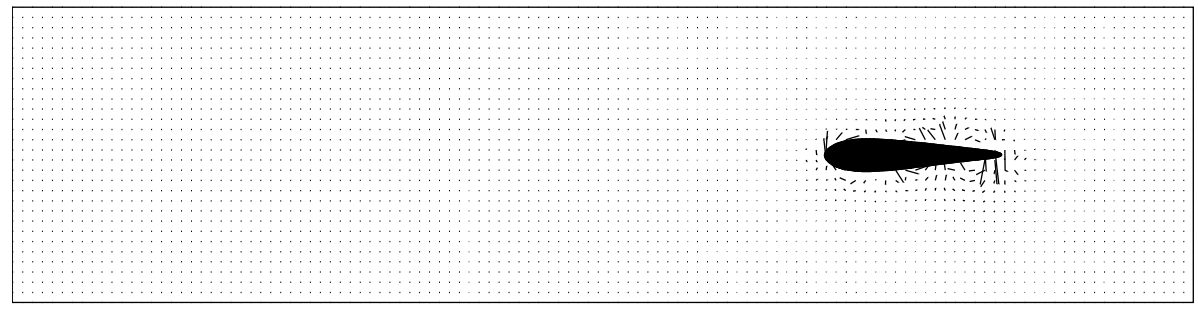

$\mathrm{t}=18.75 \mathrm{~s}$

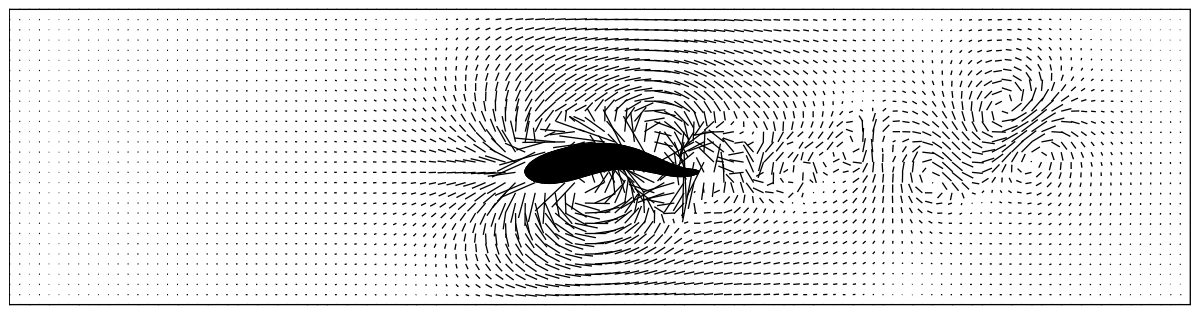

$t=37.5 s$

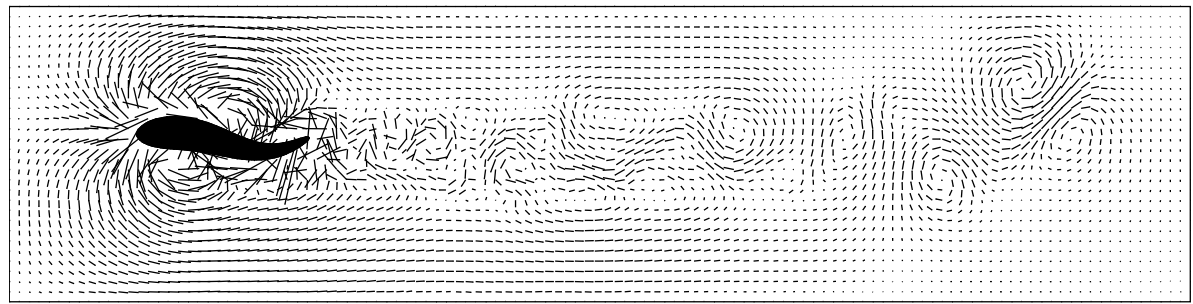

Figure 3: Straight line swimming. 


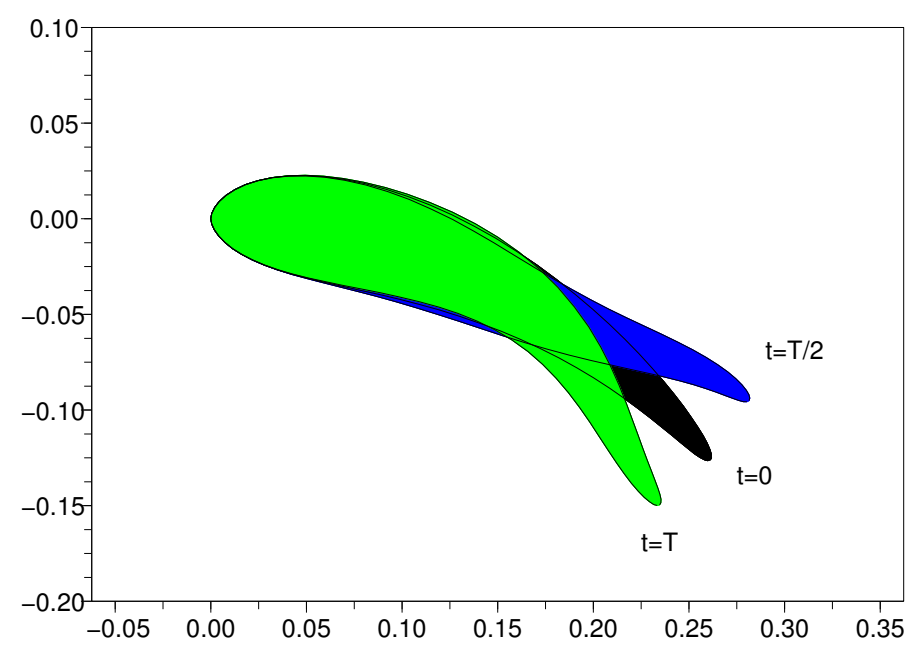

Figure 4: Deformation of the creature for the turning. 

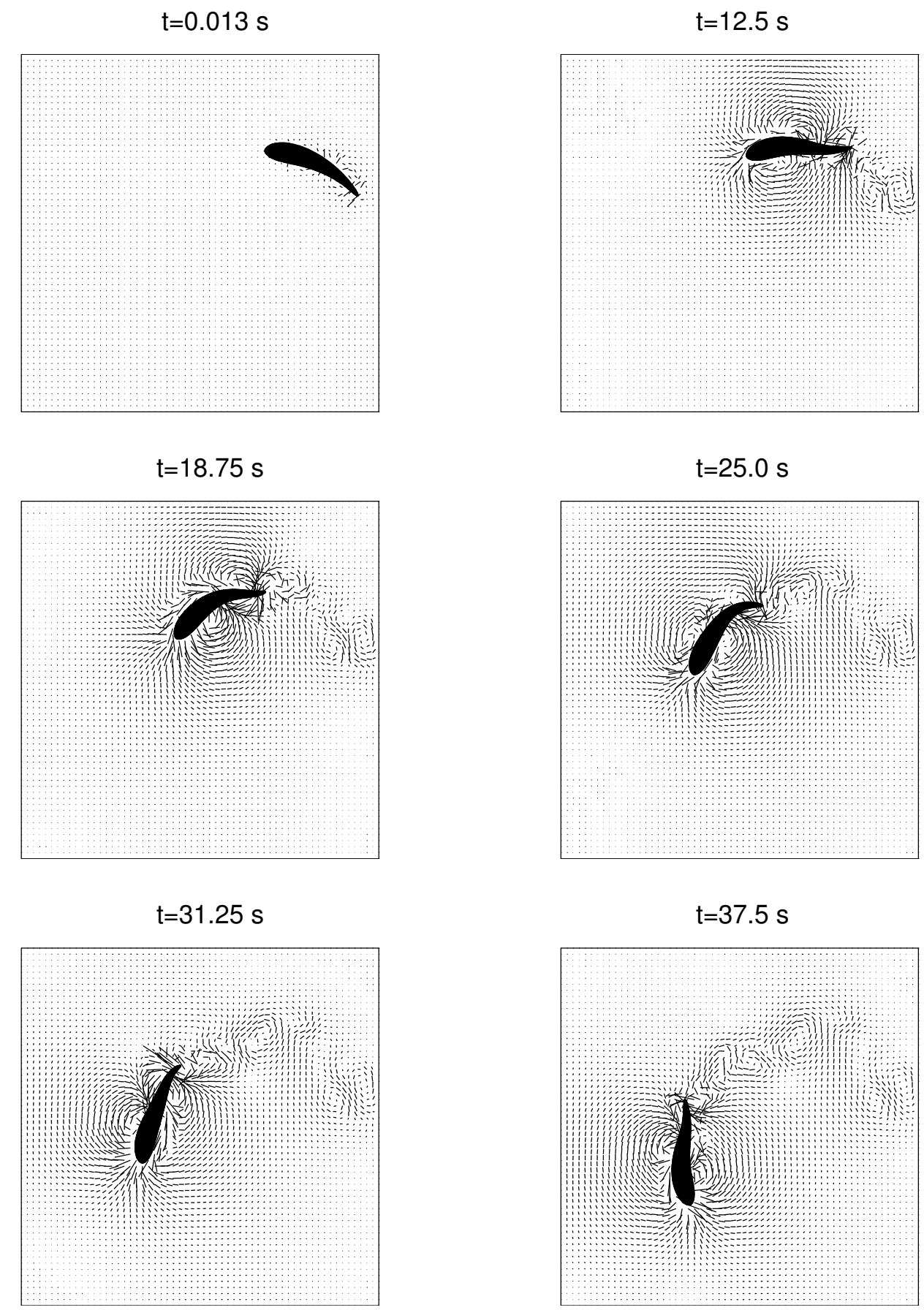

Figure 5: Turning. 
$t=0.013 \mathrm{~s}$

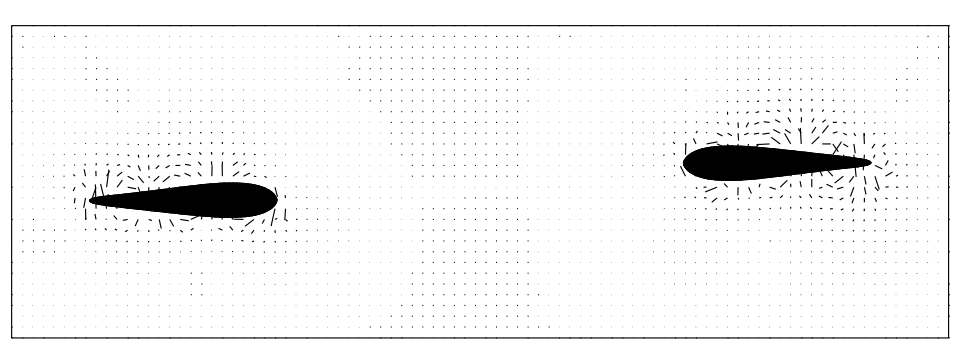

$t=9.375 \mathrm{~s}$

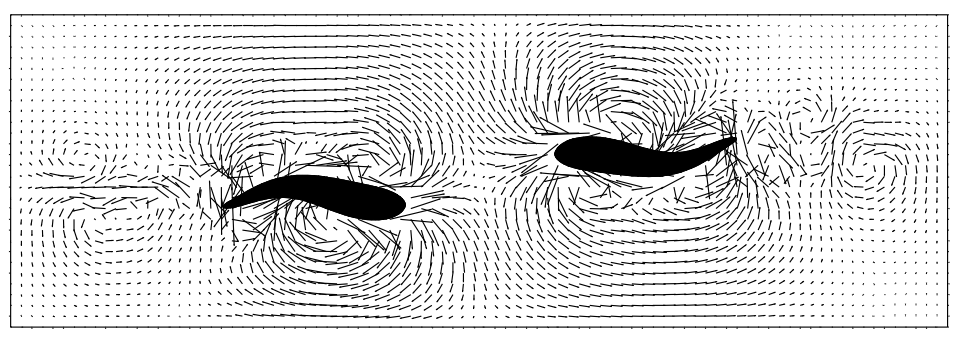

$t=14.062 \mathrm{~s}$

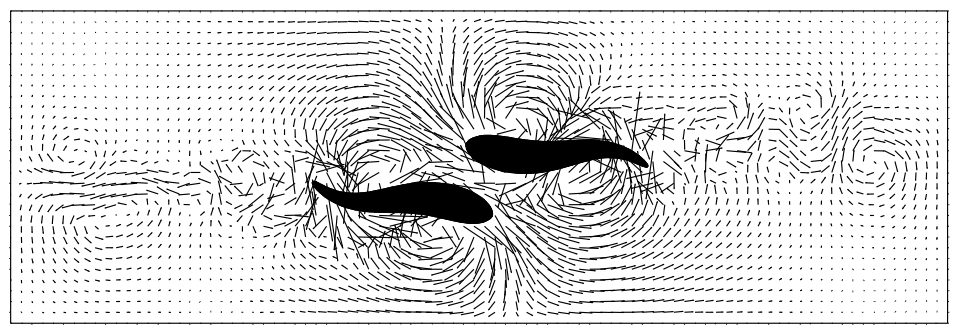

$t=20.0 \mathrm{~s}$

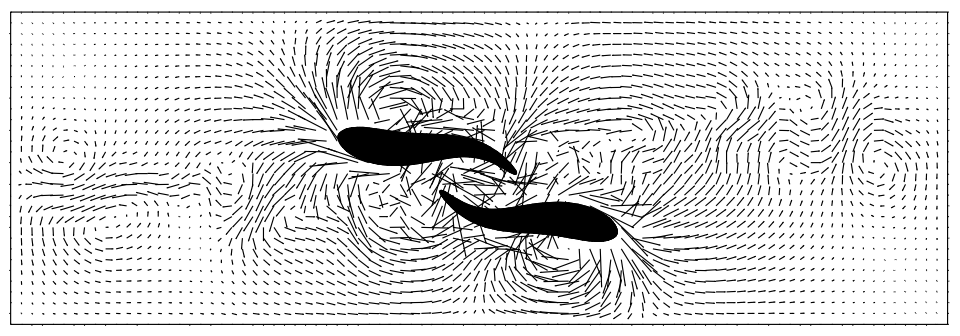

$t=25.0 \mathrm{~s}$

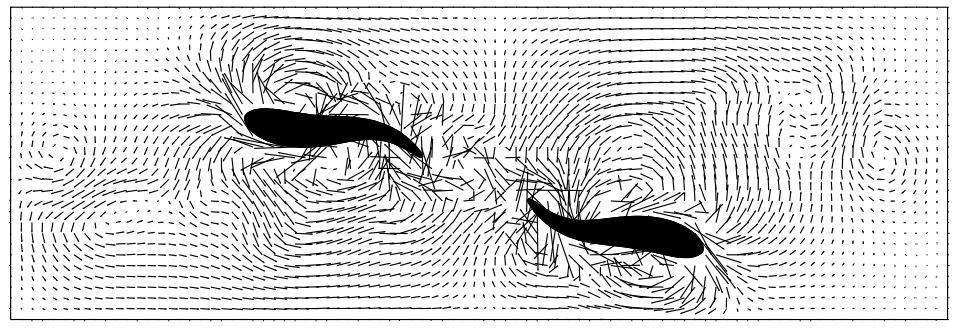

Figure 6: Meeting. 


\section{References}

[1] G. Borelli, On the movement of animals, Springer, Springer-Verlag, New York, 1989.

[2] K. Boukir, Y. Maday, B. Métivet, and E. Razafindrakoto, A high-order characteristics/finite element method for the incompressible Navier-Stokes equations, Internat. J. Numer. Methods Fluids, 25 (1997), pp. 1421-1454.

[3] M. Boulakia, Existence of weak solutions for the motion of an elastic structure in an incompressible viscous fluid., C. R., Math., Acad. Sci. Paris, 336 (2003), pp. 985-990.

[4] J. Carling, T. Williams, and G. Bowtell, Self-propelled anguilliform swimming: simultaneous solution of the two-dimensional Navier-Stokes equations and Newton's laws of motion, J.of Experimental Biology, 201 (1998), pp. 3143-3166.

[5] A. Chambolle, B. Desjardins, M. J. Esteban, and C. Grandmont, Existence of weak solutions for the unsteady interaction of a viscous fluid with an elastic plate., J. Math. Fluid Mech., 7 (2005), pp. 368-404.

[6] S. Childress, Mechanics of swimming and flying, vol. 2 of Cambridge Studies in Mathematical Biology, Cambridge University Press, Cambridge, 1981.

[7] D. Coutand and S. Shkoller, Motion of an elastic solid inside an incompressible viscous fluid., Arch. Ration. Mech. Anal., 176 (2005), pp. 25-102.

[8] — The interaction between quasilinear elastodynamics and the Navier-Stokes equations., Arch. Ration. Mech. Anal., 179 (2006), pp. 303-352.

[9] P. Cumsille and T. Takahashi, Wellposedness for the system modelling the motion of a rigid body of arbitrary form in an incompressible viscous fluid, preprint.

[10] P. Cumsille And P. Tucsnak, Wellposedness of the Navier-Stokes flow in the exterior of the rotating obstacle, Mathematical Methods in the Applied Sciences, 29 (2006), pp. 595-623.

[11] B. Desjardins, M. Esteban, C. Grandmont, and P. Le Tallec, Weak solutions for a fluid-elastic structure interaction model., Rev. Mat. Complut., 14 (2001), pp. 523-538.

[12] B. Desjardins And M. J. Esteban, Existence of weak solutions for the motion of rigid bodies in a viscous fluid, Arch. Ration. Mech. Anal., 146 (1999), pp. 59-71.

[13] E. FeIREISL, On the motion of rigid bodies in a viscous incompressible fluid., J. Evol. Equ., 3 (2003), pp. 419-441.

[14] G. GALDI, On the steady self-propelled motion of a body in a viscous incompressible fluid, Arch. Ration. Mech. Anal., 148 (1999), pp. 53-88.

[15] G. P. GALDI, On the motion of a rigid body in a viscous liquid: a mathematical analysis with applications, in Handbook of mathematical fluid dynamics, Vol. I, NorthHolland, Amsterdam, 2002, pp. 653-791. 
[16] J. Gray, Study in animal locomotion IV-the propulsive powers of the dolphin, Journal of experimental biology, 10 (1932), pp. 192-199.

[17] J. GRAY, Study in animal locomotion I-the movement of a fish with special reference to the eel, Journal of experimental biology, 13 (1936), pp. 88-104.

[18] M. Gunzburger, H.-C. Lee, And G. Seregin, Global existence of weak solutions for viscous incompressible flows around a moving rigid body in three dimensions, J. Math. Fluid Mech., 2 (2000), pp. 219-266.

[19] A. Inoue And M. Wakimoto, On existence of solutions of the Navier-Stokes equation in a time dependent domain, J. Fac. Sci. Univ. Tokyo Sect. IA Math., 24 (1977), pp. 303-319.

[20] E. Kanso, J. E. Marsden, C. W. Rowley, and J. B. Melli-Huber, Locomotion of articulated bodies in a perfect fluid, J. Nonlinear Sci., 15 (2005), pp. 255-289.

[21] S. D. Kelly and R. M. Murray, Modelling efficient pisciform swimming for control, Internat. J. Robust Nonlinear Control, 201 (1998), pp. 3143-3166.

[22] O. Ladyzhenskaya, The mathematical theory of viscous incomprehensible flow, Gordon and Breach Science Publishers, New York, 1969.

[23] A. Leroyer And M.Visonneau, Numerical methods for ranse simulations of a selfpropelled fish-like body, Journal of Fluids and Structures, 20 (2005), pp. 975-991.

[24] J. Lighthill, Mathematical biofluiddynamics, Society for Industrial and Applied Mathematics, Philadelphia, Pa., 1975.

[25] J.-L. Lions and E. Magenes, Non-homogeneous boundary value problems and applications. Vol. I, Springer-Verlag, New York, 1972. Translated from the French by P. Kenneth, Die Grundlehren der mathematischen Wissenschaften, Band 181.

[26] H. LiU AND K. KaWACHI, A numerical study of undulatory swimming swimming, J.of Computational physics, 155 (1999), pp. 223-247.

[27] B. Maury and R. Glowinski, Fluid-particle flow: a symmetric formulation, C. R. Acad. Sci. Paris Sér. I Math., 324 (1997), pp. 1079-1084.

[28] J. San Martín, J.-F. Scheid, T. Takahashi, and M. Tucsnak, Convergence of the Lagrange-Galerkin method for the equations modelling the motion of a fluid-rigid system, SIAM J. Numer. Anal., 43 (2005), pp. 1536-1571.

[29] J. San Martín, V. Starovoitov, and M. Tucsnak, Global weak solutions for the two-dimensional motion of several rigid bodies in an incompressible viscous fluid, Arch. Ration. Mech. Anal., 161 (2002), pp. 113-147.

[30] J. A. Sparenberg, Survey of the mathematical theory of fish locomotion, J. Engrg. Math., 44 (2002), pp. 395-448.

[31] T. Takahashi, Analysis of strong solutions for the equations modeling the motion of a rigid-fluid system in a bounded domain, Adv. Differential Equations, 8 (2003), pp. 1499-1532. 
[32] G. TAYLOR, Analysis of the swimming of long and narrow animals, Proc. R. Soc. London A, 214 (1952).

[33] R. Temam, Problèmes mathématiques en plasticité, Gauthier-Villars, Montrouge, 1983.

[34] M. Tryantafyllou and G. Tryantafyllou, An efficient swimming machine, Scientific american, 272 (1995), pp. 64-70.

[35] T. Y. Wu, Mathematical biofluiddynamics and mechanophysiology of fish locomotion, Math. Methods Appl. Sci., 24 (2001), pp. 1541-1564. Biofluiddynamics. 